\title{
The research of laryngeal joints to reconstruction and modeling
}

\author{
Yi Zhang and Tingchun Shi* \\ Research Center for Bio-Manufacturing, Hangzhou Dianzi University, Hangzhou, Zhejiang 310037, \\ China
}

\begin{abstract}
Larynx has a complex structure with joints and multiple functions. In order to study the artificial larynx and artificial auricle scaffold, a three-dimensional digital model of laryngeal joint is established in this paper using MIMICS with its biomechanical properties analyzed and calculated by using the finite element method. This model is based on the CT scanned images of 281 layers with an interlamellar spacing of $1.25 \mathrm{~mm}$. The obtained data are denoised, segmented and smoothed before being loaded into MIMICS. By further optimizations, an accurate and complete 3D model can be obtained. Subsequently, a 3D FEM of the normal larynx joint is performed which allows observations from any dimensions and angles. Compared with natural laryngeal joint, this model has good geometric similarity and mechanically similar throat voicing functions.
\end{abstract}

Keywords: laryngeal joints, finite element, laryngeal reconstruction, three-dimensional reconstruction

\section{Introduction}

People's vocalization, breathing and swallowing are controlled by the cricoid cartilage in laryngeal tissues. Nowadays, 136000 patients all over the world are suffering from laryngeal cancer or laryngeal trauma. Due to the limitations of medical equipment and conditions, laryngectomy is often limited to a small number of patients [1]. In laryngectomy treatment, normal cartilage tissues will be removed, during which the large resection might cause secondary trauma [2]. However, with the development of tissue engineering and in-depth research of additive manufacturing (AM), a new method in biomedical engineering which uses the scaffold of AM to graft and substitute defect tissues has been developed to repair tissues and transplanting organs. In tissue engineering, the merits of scaffold structure play an important role in the physiological functions of organs and tissues for recovery. Besides, the tissue scaffold fabrication is implemented solely using AM technology. As a result, it is of great importance to establish a three-dimensional model of cricoid cartilage to investigate the rehabilitation of laryngeal cartilage injuries. Based on the study of early artificial ear and artificial spinal cord in tissue engineering, three steps are required to establish a precise three-dimensional model of cricoid cartilage and obtain biomechanics of cricoid cartilage using finite element analysis (FEA). First, the larynx model should be extracted from the gray value of CT image data obtained from the neck of a healthy

*Corresponding author: Tingchun Shi, Research Center for Bio-Manufacturing, Hangzhou Dianzi University, Hangzhou, Zhejiang 310037, China. Tel.: 13216702816; Fax: 057187713528; E-mail: stc@hdu.edu.cn.

0959-2989/14/\$27.50 @ 2014 - IOS Press and the authors. 
subject. Second, the model of cricoid cartilage needs to be isolated from the entire larynx model. Third, biocompatible materials need to be provided to the model of cricoid cartilage with finite element analysis [3].

\section{Materials and methods}

\subsection{Equipment and software}

Equipment: Computer- 2G memory, Windows XP, hard drive 250G, 14-inch LCD.

Software: MIMICS 10.01(Belgium), ANSYS (USA).

\subsection{CT images collection}

The CT neck image data of a healthy subject (man, 29 years old) are obtained from Hangzhou Plastic Surgery Hospital by spiral CT scan. We pay attention to two aspects of the CT scan in order to fulfil the requirements of subsequent model establishment Firstly, the subject's head needs to maintain steady in vertical orientation when being scanned for CT slice once every $1.25 \mathrm{~mm}$ pitch [4]. Secondly, as only the CT data between nostrils and should are needed, the scan starts below the nose, which could save subsequent modeling time. Data obtained from CT scan is saved in DICOM format, which can be loaed into MIMICS for subsequent processing.

\subsection{Establishment of the three-dimensional model}

Using MIMICS, image enhancement and region growing segmentation for laryngeal tissue are firstly applied to CT images obtained [5,6]. Threshold segmentation is subsequently applied to these images, converting grayscale images into binary ones [7,8]. Finally, each image is individually processed to remove redundant data using edge segmentation, selective editing, and noise removal [9-12].

The model of the laryngeal cartilage is further divided into thyroid cartilage, cricoid cartilage and joint. As shown in Figure 1, (a) includes the thyroid cartilage, cricoid cartilage and joint, (b) includes the thyroid cartilage only and (c) includes the cricoid cartilage only.

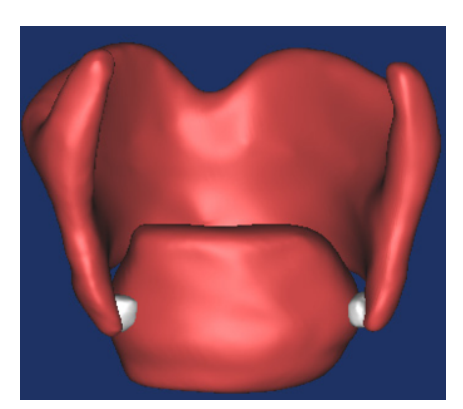

(a) Cartilage

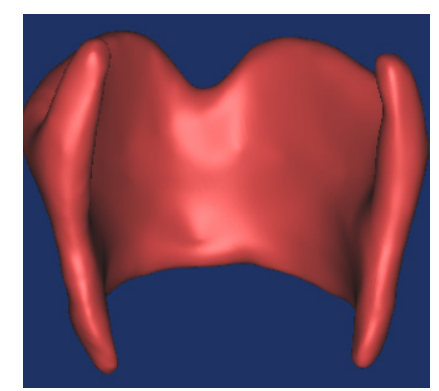

(b) Thyroid cartilage

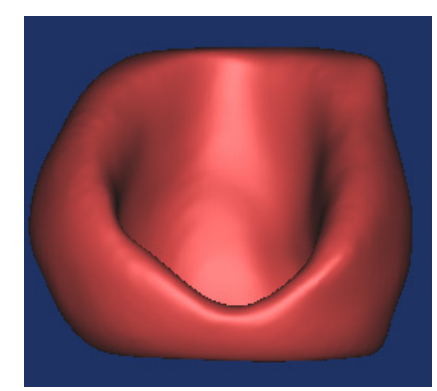

(c) Cricoid cartilage

Fig. 1. Thyroid cartilage and cricoid cartilage models. 


\section{Finite element analysis}

The annular cricoid locates at the bottom of the larynx, between the cricoid arch and the cricoid plate. The cricoid pressure $(\mathrm{CP})$ is a standard of practice for general anesthesia induction adopted on full-stomach patients undergoing an emergency operation. It is defined as a method in which CP will prevent regurgitation of gastric contents into the pharynx at esophageal pressures so that aspiration pneumonia can be avoided after anesthesia [13-15]. Vanner recommended that a reasonable cricoid pressure should be $10 \mathrm{~N}$ for awake patients at first, and then increase to $30 \mathrm{~N}$ after patients become unconscious [16,17].

Thyroid cartilage includes a left and a right cartilage plate, which protects the throat and is also susceptible to injuries due to natural and man-made violence.

\subsection{Optimization of the surface mesh model}

The FEA module of MIMICS can quickly process the input scanned data and output the data in an appropriate file format for FEA. Users can load the scanned data to create 3D models which can then be meshed for FEA [18]. The "remesh" command in MIMICS optimizes 3D models to improve the triangle qualities. The "magics" command can detect and remove crosses and poor quality triangles for mesh, which could simplify the models and reduce the workload of FEA [19].

An unmeshed model is shown in Figure 2(a) and its surface mesh quality unit needs to be tested before being meshed. The "standard" unit is the ratio between the length of the height and the side of the triangle (H/B) with a value of 0.25 . The colored triangles in Figure 2(b) are triangular pieces with a value less than 0.3 mass, which fails to meet $19 \%$ of the triangle requirements, the others are unqualified.

In Figure 3, (a) shows the meshed model and (b) shows its quality test histogram. As shown in Figure 3(b), triangles with a one-sided unit of low quality standard have been completely removed and the quality of the surface mesh has been standardized. Eventually, the quality of the generated volume mesh is improved while the number of triangles is reduced from 186467 to 8380 , which could save the calculation time and improve the credibility of the result.

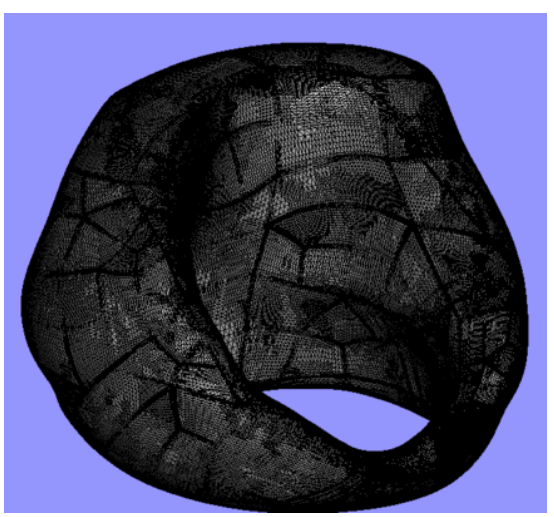

(a) Primitive mesh model

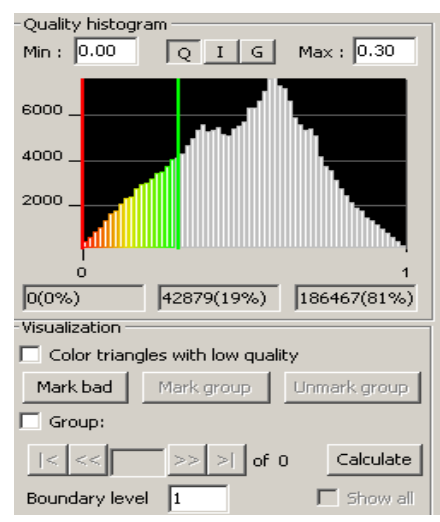

(b) Quality testing histogram

Fig. 2. Non-optimized mesh model. 


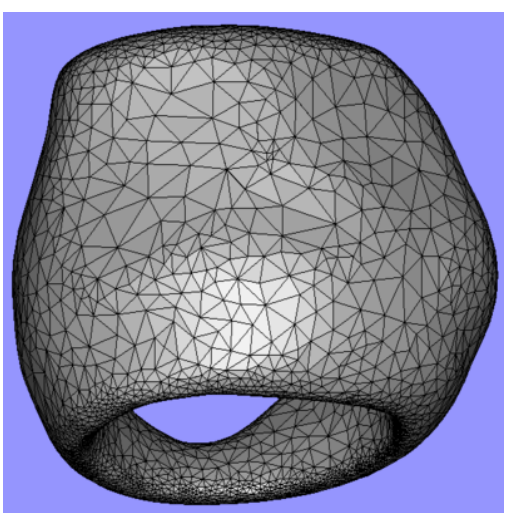

(a) Primitive mesh model

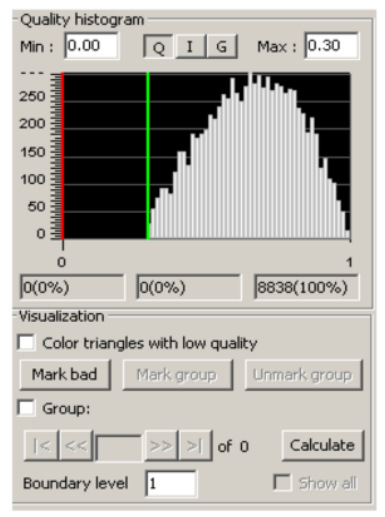

(b) Quality testing histogram

Fig. 3. Optimized mesh model.

To summarize, meshing is perform to improve the quality of triangles. In MIMICS, the 3D model is meshed and optimized using the "remesh" command of the FEA module. The ".lis" format file is also exported using the "remesh" which can be subsequently used to create volume mesh model in ANSYS $[20]$.

\subsection{Surface mesh model into volume mesh model}

The ".lis" format file is imported into ANSYS to create a volume mesh model as shown in Figure 4, where the nodes and elements are further reduced when the solid92 unit is selected.

\subsection{Finite element analysis of the cricoid model}

A finite element model of the cricoid cartilage is analyzed with load using the ANSYS finite element system (ANSYS10.0).

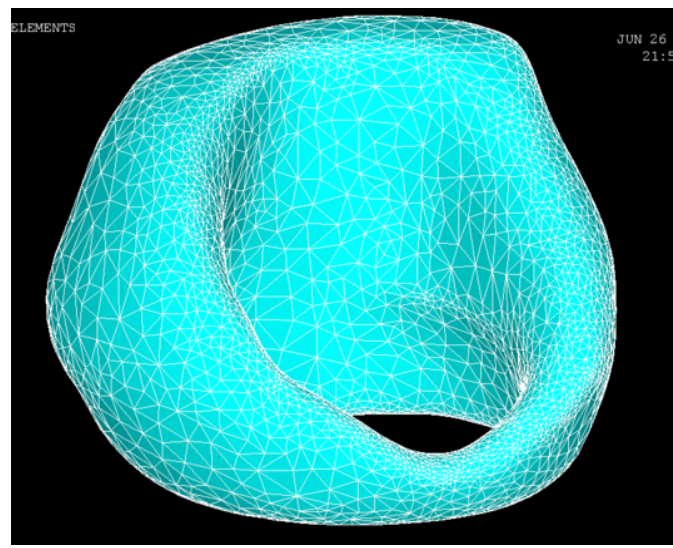

Fig. 4. Volume mesh model.

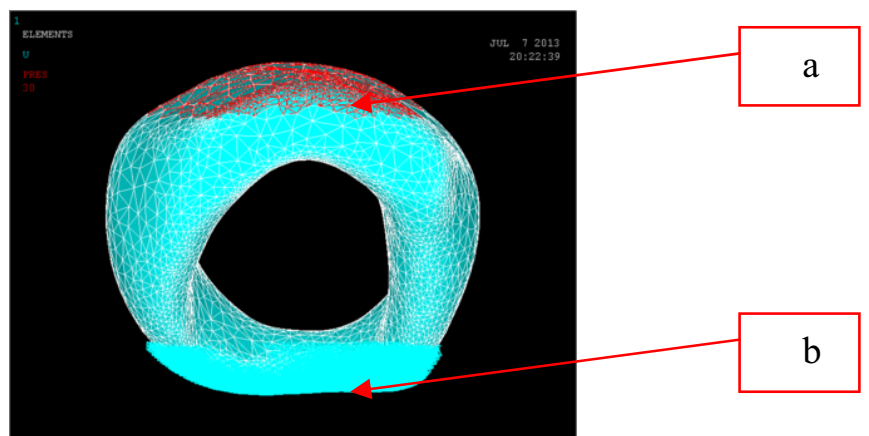

(a) Measured parameters for a $30 \mathrm{~N}$ load $\quad$ (b) Constraints side.

Fig. 5. Loading force diagram. 


\subsubsection{Material settings}

The nonlinearity, anisotropy and heterogeneity are mechanical properties of laryngeal cartilage and the 3D model of characteristics can be simplified when mechanical analysis is implemented for the laryngeal cartilage. Cricoid is generated by isotropic, homogeneous and continuous linear elastic materials with an elastic module of around 60 MPA, and a Poisson ratio of 0.47 [21].

\subsubsection{Load settings}

In this study, the force used in clinical anesthesia $\mathrm{CP}$ is adopted for adults. Along the direction of cricoid plate, $30 \mathrm{~N}$ is applied to cricoid cartilage to simulate the force of cricoid.

\subsubsection{Boundary conditions}

We fix the cricoid arch, which means the displacements of the remote nodes in $\mathrm{X}, \mathrm{Y}, \mathrm{Z}$ axis are 0 . 8000 nodes are selected for applying pressure from the central surface of cricoid plate. Based on CP simulations, cricoid arch is constraint's side as steady. $30 \mathrm{~N}$ is applied to the cricoid plate with the stress distribution presented in Figure 5.

Figure 6 gives the ANSYS calculations of the stress distribution on cricoid arch when pressure is applied. ANSYS gives von mises stress distribution, in which gradual transition of color from blue to red means the gradual increase of force and maximum stress exists in the red region.

According to Figures 5 and 6, when the cricoid arch is fixed and load is applied to the cricoid plate, stress mainly exists on the cricoid arch, especially inside and outside of cricoid arch $1 / 3$ segment. This is due to the fact that the inside of cricoid arch inside is mainly exposed to compressive stress of CP, and the outside is mainly exposed to tensile stress.

\subsection{Finite element analysis of the cartilage model}

Material settings are 20 MPA natural cartilage modules and 0.3 Poisson ratio. Artificial cartilage is prepared using polyurethane materials $[22,23]$.

Load settings, boundary conditions and fix the cartilage to one side, and the stress distribution when $100 \mathrm{~N}$ pressure is applied is given in Figure 7.

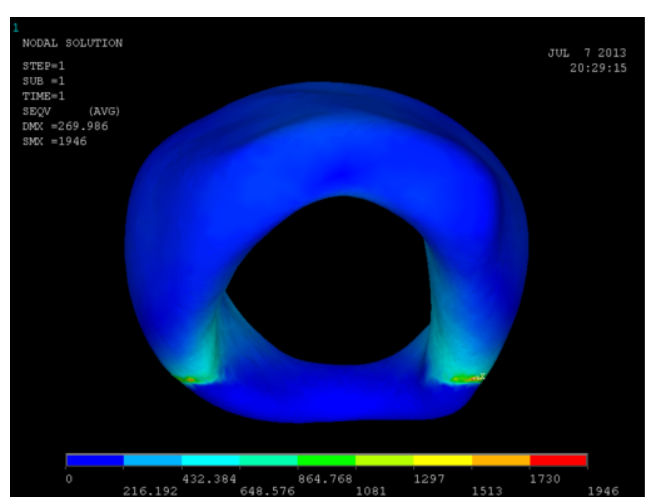

(a) Front side.

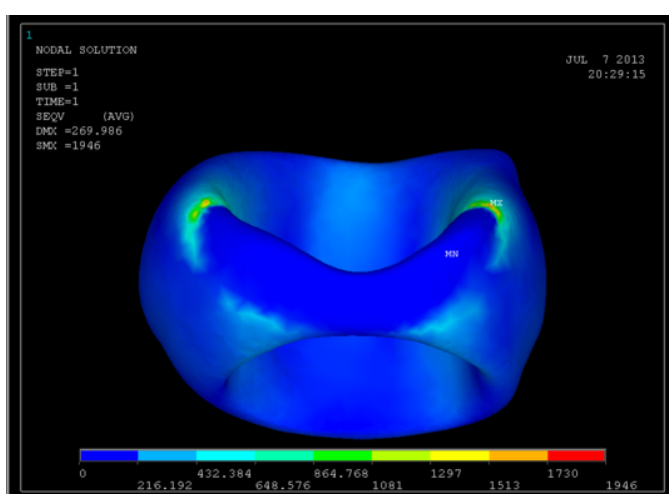

(b) Rear side.

Fig. 6. Force distribution of cricoid as calculated by ANSYS. 
For the same conditions, artificial cartilage material and natural cartilage material are used to simulate the displacement deformation. As shown in Figure 8, the maximum deformations of natural and artificial cartilage are 288568 and $121650 \mathrm{um}$, respectively.

According to the ANSYS analysis, the von mises stress distribution of thyroid cartilage is shown in Figure 9.

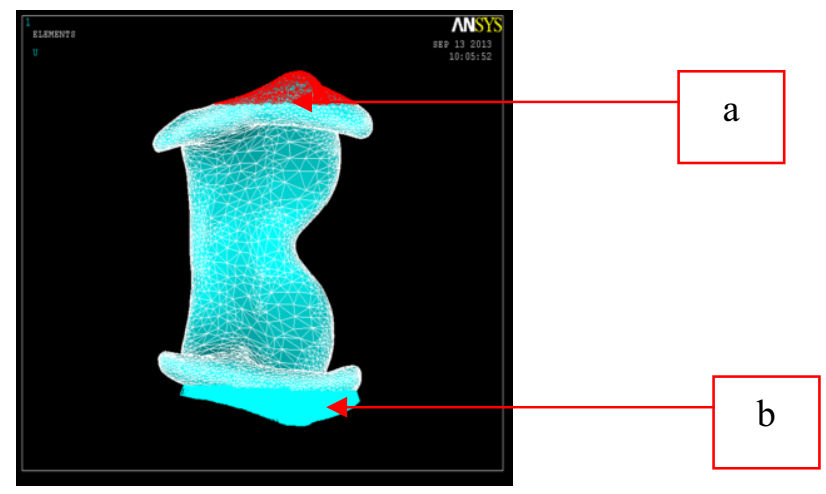

$\begin{array}{ll}\text { (a) Measured parameters for a } 100 \mathrm{~N} \text { load } & \text { (b) Constraints side }\end{array}$ Fig. 7. Loading force diagram.

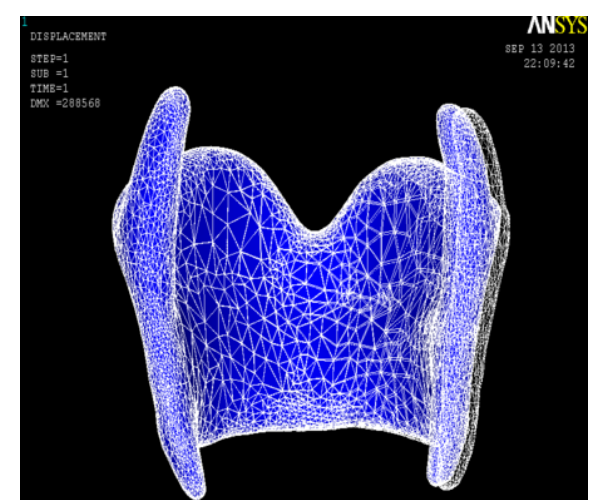

(a) Natural cartilage.

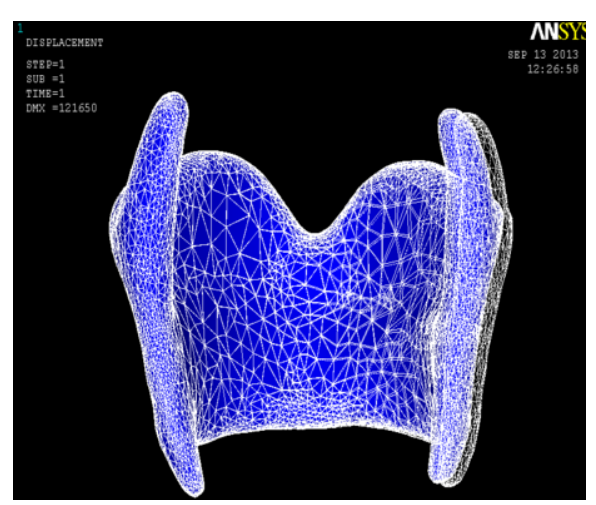

(b) TPU artificial cartilage.

Fig. 8. Diagrams of thyroid cartilage.

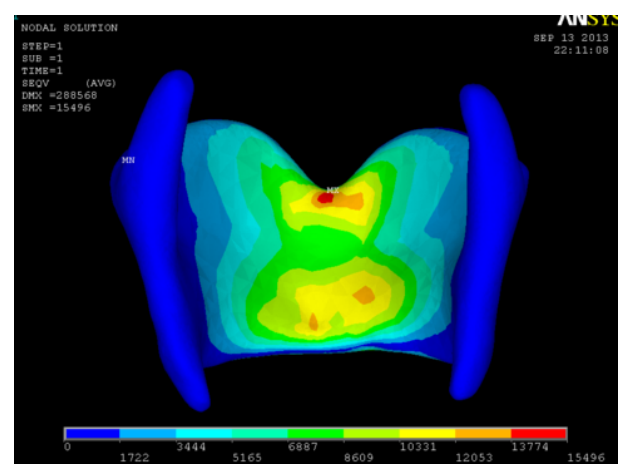

(a) Natural cartilage.

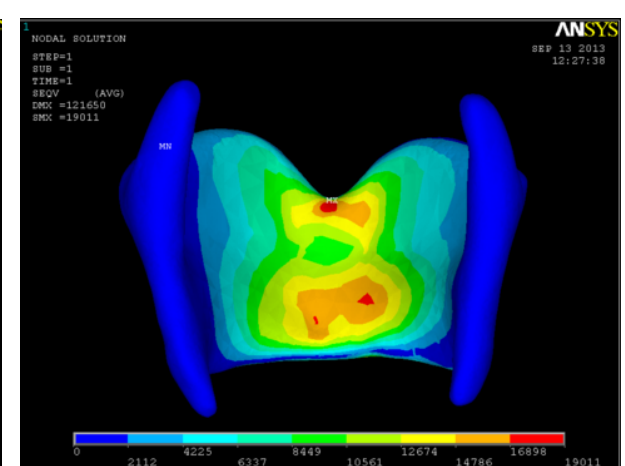

(b) TPU artificial cartilage.

Fig. 9. Force distribution of thyroid cartilage. 
According to Figures 8 and 9, when the left and right sides of the thyroid cartilage plate are squeezed, load stress mainly concentrates inside the plate and the major points for natural and artificial cartilage are quite similar.

\section{Discussion}

CP has been used in clinical anesthesia for more than 40 years, and it has commonly used for high-risk patients who might be in the risk of aspiration during anesthesia. Due to its safety and effectiveness, CP is drawing more and more attention from the anesthesiologists [24].

In this study, the MIMICS software is used to process CT images to establish a three-dimensional digital model of laryngeal cartilage tissue, which acts as a visualization platform for clinical diagnostics and medical educations. The data from the digital model can be directly converted the format suitable for finite element analysis, which facilitates further biomechanical research and applications. The 3D model is perfect in morphological and structural similarity. The establishment of digital models using this method has a good repeatability which allows extension into three-dimensional digital models of other parts of the body [25]. In this study, we only establish three-dimensional models for the cricoid and thyroid cartilage and corresponding mechanical test and finite element stress analysis are completed. To summarize, this paper has laid a solid foundation for the subsequent 3D scaffold printing with materials and future studies of medical experiments.

\section{Acknowledgement}

This study is supported by the National Natural Science Foundation of China (61272389).

\section{References}

[1] M.L. Ng, C.L. Kwok and S.F. Chow, Speech performance of adult cantonese-speaking laryngectomees using different types of alaryngeal phonation, Journal of Voice 11 (1997), 338-344.

[2] M.J. Russell, D.M. Rubin, B. Wigdorowitz et al., The artificial larynx: A review of current technology and a proposal for future development, in: 14th Nordic-Baltic Conference on Biomedical Engineering and Medical Physics, Springer, Berlin Heidelberg, 2008, pp. 160-163.

[3] P. Li, R. Liang, D.L. Shen et al., Establishment of three-dimensional finite element model of human mandible with MIMICS and ANSYS software, Journal of Practical Stomatology 28 (2012), 709-713.

[4] Y.B. Li, B. Hong, S.K. Gao et al., Bone segmentation in human CT images, Journal of Biomedical Engineering 21 (2004), 169-173.

[5] N.S. Nusinov and W.D. Gay, A method for obtaining the reverse image of an ear, The Journal of Prosthetic Dentistry 44 (1980), 68-71.

[6] T.J. Coward, A comparison of prosthetic ear models created from data captured by computerized tomography, magnetic resonance imaging, and laser scanning, International Journal of Prosthodontics 20 (2007), 275-285.

[7] C.R. Crawford and K.F. King, Computed tomography scanning with simultaneous patient translation, Med. Phys. 17 (1990), 967-982.

[8] C.Y. Bao and J.H. Liu, Inverse engineering research on lumbar segment of human body vertebral column based on CT images, Journal of Machine Design 9 (2008), b16-18.

[9] K. Subburaj, C. Nair, S. Rajesh et al., Rapid development of auricular prosthesis using CAD and rapid prototyping technologies, International Journal of Oral and Maxillofacial Surgery 36 (2007), 938-943.

[10] T. Sandor, D. Metcalf and Y.J. Kim, Segmentation of brain CT images using the concept of region growing, International Journal of Bio-Medical Computing 29 (1991), 133-147. 
[11] J. Zhang and C.H. Yan, Fast segmentation of bone in CT images using 3D adaptive thresholding, Computers in Biology and Medicine 40 (2010), 231-236.

[12] E.L. Hartsilver, R.G. Vanner, J. Bewley et al., Gastric pressure during emergency caesarean section under general anaesthesia, British journal of Anaesthesia 82 (1999), 752-754.

[13] R.G. Vanner, J.P. Dwyer, B.J. Pryle et al., Upper oesophageal sphincter pressure and the effect of cricoid pressure, Anaesthesia 47 (1992), 95-100.

[14] R.G. Vanner and B.J. Pryle, Regurgitation and oesophageal rupture with cricoid pressure: A cadaver study, Anaesthesia 47 (1992), 732-735.

[15] M.J. Ruth and R. Griffiths, Safe use of cricoid pressure, Anaesthesia 54 (1999), 498-498.

[16] H. Yu and Y.Q. Wang, Clinical application for cricoid pressure, West China Medical Journal 17 (2002), $432-434$.

[17] L.Z. Wang, CT scan based finite element analysis of lumbar vertebral body, Ph.D. Dissertation, Jilin University, 2007.

[18] P.F. Jiao, D. Qi and Z.L. Qi, Virtual mandibular angle reducation plasty with a 3D model based on CT data, Acta Anatomica Sinica 37 (2006), 449-451.

[19] R. Nareliya and V. Kumar, Biomechaical analysis of human femur bone, International Journal of Engineering Science \& Technology 3 (2011), 312-317.

[20] M.X. Liu, H.J. Liu and W.M. Yu, Polyurethane Elastomer Manual, Chemical Industry Press, Beijing, 2001.

[21] J.K. Suh, I. Youn and F.H. Fu, An in situ calibration of an ultrasound transducer: A potential application for an ultrasonic indentation test of articular cartilage, Journal of Biomechanics 34 (2001), 1347-1353.

[22] X.C. Wei, R.J. He, Z.Q. Yang et al., Preparation and physical properties of cellular cartilage material, China Journal of Experimental Surgery 26 (2009), 511-513.

[23] X.C. Xu, Y.W. Wang, H. Kang et al., The measurement of elastic modulus of normal cadacer TMJ cartilage, Chinese Journal of Prosthodontics March 1 (2000), 14-17.

[24] T. Asai, K. Barclay, C. McBeth et al., Cricoid pressure applied after placement of the laryngeal mask prevents gastric insufflation but inhibits ventilation, British Journal of Anaesthesia 76 (1996), 772-776.

[25] D. Fu, A.M. Jin, S.X. Min et al., A digital model for lumbar motion segment reconstruction and three-dimensional visualization, Journal of Southern Medical University 27 (2007), 1376-1378. 